\title{
Designing Simplified Exergame for Muscle and Balance Training in Seniors: A Concept of 'Out in Nature'
}

\author{
Ather Nawaz ${ }^{1}$, Mathilde Waerstad ${ }^{2}$, Kine Omholt ${ }^{2}$, Jorunn L. Helbostad ${ }^{1}$, Beatrix Vereijken ${ }^{1}$, \\ Nina Skjæret ${ }^{1}$ and Lill Kristiansen ${ }^{2}$ \\ ${ }^{1}$ Department of Neuroscience, ${ }^{2}$ Department of Telematics \\ Norwegian University of Science and Technology (NTNU), Norway \\ ${ }^{1}$ \{ather.nawaz, jorunn.helbostad, nina.skjaret, beatrix.vereijken\}@ntnu.no \\ ${ }^{2}$ lillk@item.ntnu.no, ${ }^{2}$ mathildewaerstad, kine.omholt\}@gmail.com
}

\begin{abstract}
If exergames are to be used successfully to improve physical function in senior citizens, they need to be designed specifically for this target group. Muscle strength and balance training are the key components of exercise to prevent falling in seniors. The aim of this study was to design a new, simplified exergame concept for senior citizens, which focuses on physical activity in general, and balance and muscle strength in particular. The study provides a new design concept of an exergame on the basis of assessment of 7 senior participants' $(70.6 \pm 7.9$ years $)$ experience with three commercially available exergames. The participants stated that the design of the existing exergames should be simplified and closely related to activities that can be associated with their daily life. The feedback was qualitatively analyzed to enlist the requirements of a new exergame. A new simplified exergame design concept, Out in Nature, was created on the basis of the seniors' feedback. The new design focused on a simplistic design, playing games for balance and muscle training related to real life activities. The study demonstrates a concept for a game developed for seniors that can be used to train aspects of balance and gait as part of natural tasks.
\end{abstract}

\section{Keywords}

Exergames, Design, Seniors, Balance, Falls

\section{INTRODUCTION}

The performance of exercise through video games is known as exergames. The use of exergames is increasingly popular among young people. Most of the commercial exergames are mainly designed for children and adolescents for the purpose of entertainment. The game industry is growing $9.1 \%$ annually to \$48.9 in 2011 and \$66 billion in 2012, making it the fastestgrowing component of the international media. Due to business goals, the game industry is mainly focusing on covering a large population without specific focus on the requirements of senior citizens.

The world's population is "greying", with the proportion of the

Permission to make digital or hard copies of all or part of this work for personal or classroom use is granted without fee provided that copies are not made or distributed for profit or commercial advantage and that copies bear this notice and the full citation on the first page. To copy otherwise, to republish, to post on servers or to redistribute to lists, requires prior specific permission and/or a fee.

REHAB 2014, May 20-23, Oldenburg, Germany

Copyright (c) 2014 ICST 978-1-63190-011-2

DOI 10.4108/icst.pervasivehealth.2014.255269 world's population aged 60 and older set to double to more than $22 \%$ of the overall population by 2050 [1]. The ageing process includes decline in visual and auditory systems and slowing down in movements [2]. Due to the increasing number of older people, movement impairments and the lack of physical activity among older people will become a societal challenge in the next decades. One in three persons over the age of 65 and half of those over the age of 80 fall at least once per year. International guidelines on physical activity in older people highlight the need for balance and muscle strength training in order to prevent loss of physical function and to prevent falls [3].

Thus, there is an interest to get older people to exercise more regularly. Exergames have the potential to motivate senior citizens to be physically active. Exergames also have the potential to improve health-related issues among senior citizens, such as decreasing depression [4] and increasing physical function in general [5] and balance in particular [6]. In order to make older people to want to use exergames, the games need to be developed based on the older persons' needs and preferences. In order to prevent physical decline and falls, exergames developed for older persons should also train their balance and muscle strength.

In this paper, we evaluate existing exergames and present the design case of a new exergame "Out in Nature". The key objective is to develop a simplified concept of an exergame for senior citizens using natural tasks that provide an opportunity to train aspects of balance and muscle strength. On the basis of workshops, the study highlights challenges for designing exergames.

\subsection{Exergame design for seniors}

In developing the exergame we employed a user-centered design (UCD) which is an iterative process that keeps the users of the system in the center. Particular needs of seniors should be gathered through user-centered design process to make effective exergames for seniors [7].

To meet individual variability in the skill level of seniors, games should provide different levels so seniors can adjust according to their requirements. Gerling et al. [8] outlined four general points that are important for designing exergames for senior citizens which include the possibility to play both sitting and standing, avoid too extensive and sudden movements, adjust difficulty level, adjust device sensitivity, simple interaction mechanisms and constructive feedback in the gameplay. In order to improve balance, weight shift during movement may also be important [9]. Social interaction during the game playing can be another important factor and motivator for senior citizens. 


\section{METHOD}

Within the user-centered design approach for designing exergames for seniors, this study employed user testing, prototypes, and focus groups [7]. Two workshops were held with seniors. In the first workshop, senior citizens played three commercially available exergames and gave feedback about the game experience. Then, a prototype of a new exergame was designed on the basis of the feedback from the first workshop. A second workshop was then held to evaluate the concept of the new exergame and get seniors' feedback on the prototype of the exergame.

\section{WORKSHOP 1 - TESTING COMMERCIAL EXERGAMES}

The first workshop aimed to understand the relationship between seniors, video games and exercising, in addition to observe how elderly interact with existing video games aimed at exercising. The workshop consisted of a gameplay section followed by a group discussion where participants freely discussed their experience of commercially available exergames, their thoughts about the exergames and the technology, and their requirements for a new exergame. Senior citizens were recruited from a senior citizen organization, the Senior network, that aims at making elderly citizens active users of ICT and Internet. A total of seven senior citizens $(70.6 \pm 7.9), 4$ females and 3 males, agreed to participate in the workshop. All the participants were physically and mentally fit for their age. The workshop was held over two days, 65 minutes each, where 4 senior citizens participated on the first day and 4 participated on the second day. The participants had no prior experience with video game technologies.

Three commercial exergames were used in the first workshop, "Kinect Sports: Season Two", "YourShape:Fitness Evolved", and "Fruit Ninja". Kinect Sports consists of six sports. We chose Tennis and Skiing because generally these games stimulate movement and balance activity in a fun and motivating way, though exercising is not the main focus. YourShape is a fitness game that includes games such as cardio boxing and yoga. We chose this game to let the seniors experience a game that is designed with exercise as the main purpose. Fruit Ninja is a game where players use arms like a ninja to slice fruit that is thrown up into the air. This game was chosen on the basis of fun and movement.

The workshop was conducted at a location that was familiar to the senior citizens. Before playing the three exergames, participants were introduced to the technology and how to interact with it. The first group of participants first played the games individually, and then competed against one another using a multi-players setting. Each participant played three games for a total of approximately 25 minutes. After gameplay, the participants provided feedback on their experience with the gameplay and the technology for all three exergames in a focus group interview. Two video cameras were used to capture facial expressions, movements, and all interaction with the games. During the interview, participants were asked about the challenges they faced while playing the exergames. A set of themes was derived from the feedback of participants in workshop 1.

\subsection{Findings in workshop 1}

In the interview, the participants expressed that they liked Tennis and Skiing because they could relate it to previous real life experiences of Skiing and Tennis. They also liked it because these two games were physically more challenging, fun and entertaining. Skiing was the game that engaged the players most while playing the game because Skiing is a popular sports in the area. Following are the general points which seniors expressed in the focus group discussion.

Information display: It was a general opinion among the seniors that there were too many elements on the screen during the gameplays of all games. For the Fruit Ninja game, one of the participants stated, there are too many elements on the screen. Where should you look? The participants stated that too many things were going on at the same time and that it was hard to do multiple hand movements simultaneously. The participants expressed that the interface was complex in all three games. One of the participants said that the menu was extremely difficult, while another stated while trying to give input through hand gestures, this is worse than working with the mouse on the computer. The participants wanted to see less information and elements at the screen at the same time.

Clear objectives: Participants demanded information regarding the specific effect of the exercises. They wanted to know why to do the certain tasks and what kind of training benefits they could get for doing exergame tasks beyond entertainment. The participants also wanted to know if the movements were done correctly or not. One of the participants stated, It [the game] needs to be softer and [there should be] more instructive introduction into the game's rules and objectives. From a design perspective, the purpose and benefits of doing the exercises should be clearly described.

Progress and learning: Participants of the workshop wanted to see a simple way to display progress and a learning curve in their game-play. One of the participants stated, It is all about the experience of mastery, which is essential. The older you get, the more important it gets to progress. One of the participants suggested different levels of tasks in the game and stated, eventually, when you get better and manage to keep track of things, you can add more elements to the game. A lot of what happens in these games is not relevant. The other participant related the progress in the game to an aerobic class, we used to attend aerobic class where they practiced exercises slowly before they could do it fast. I do not feel it the same way.

Appropriate music: The participants did not like music and background noise in the three games. Cheering, loud music, encouraging comments and fans were perceived as noisy and annoying by some. One of the participants stated, I think everything about that (music) was too much. The participants rather wanted old music of their time, with less noise. Another participant stated, I think everything about that [sound] was too much. You get sensitive to sound, you want to be active but without too much noise.

In the feedback session in workshop 1, participants suggested that real-life activities such as wood chopping, sports, swimming, rowing, picking apples, biathlon, interval exercises, dance, puzzle games, and a walk in nature are some of the real life activities they wanted to see in the game design. Participants also wished for a chance to try out the exercises slowly first. In addition, it was mentioned that if the aim of the game was exercising, they wanted the whole range of exercising, from warm-up to cooldown and stretching. 


\section{PROTOTYPING THE NEW EXERGAME}

Based on the feedback from workshop 1, a horizontal prototype exergame concept was developed. A horizontal prototype provides a broad view of the system, focusing on user interaction more than on lower-level system functionality. A horizontal prototype reduces the level of functionality and results in a user interface of the surface layer. We made a horizontal prototype because it was a quick way of designing an interface and get feedback before moving to the next phase for development of an exergame. Keeping in mind Houde and Hill's model [10] for interaction design, the prototype focused on visual and sensory experiences of exergame design.

Figure 1 provides an example of an exergame interface which was prototyped for seniors. The exergame contains different levels of difficulties. The player completes the easiest level, and then proceeds to the next level. This is done for the player to be able to learn the game sufficiently before progressing to a more advanced level. The player could also play the game in collaboration with other players or compete with other players.

In the new concept, the seniors can choose exercises according to different muscle groups and balance challenges, and choose an exercise depending upon their own preference or the recommendation from their physiotherapists. The design of the exergame shows clear goals for training muscle strength and balance. In one of the exergame concepts, the design focused on balance and muscle strength, where the task was to pick apples. This activity requires players to stretch their body to reach ripe apples (balance) and put them in a basket on the ground by

more on the actual activity rather than confusing them with rich information displayed at different places of the screen.
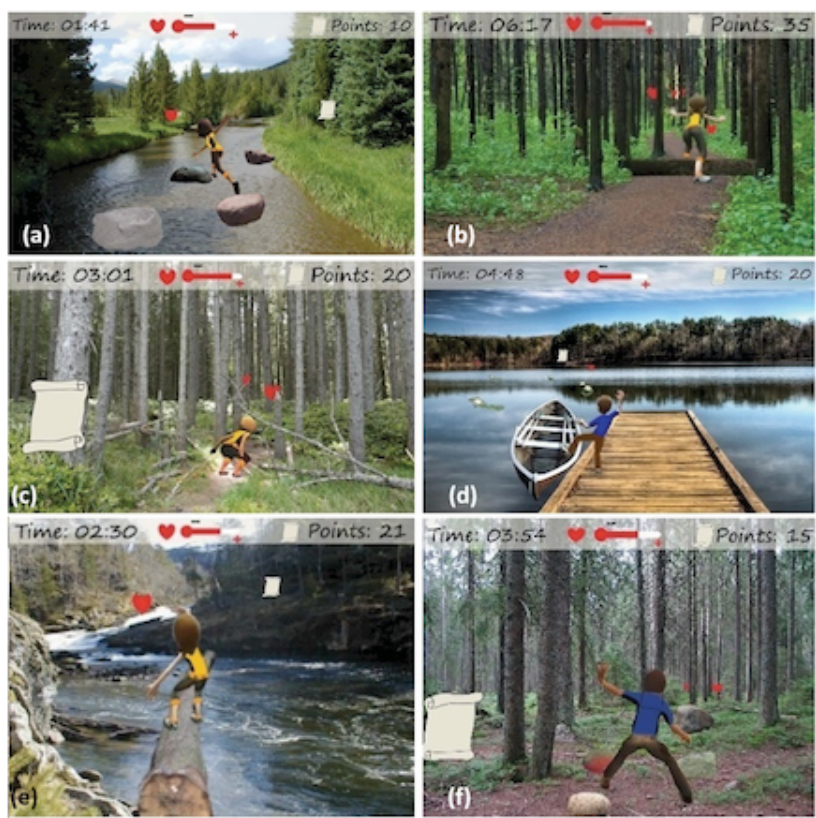

Figure 2. Obstacles in the nature trail

Figure 2 presents the interface of different obstacles in the nature trail, which challenges users' muscle strength and balance. A set

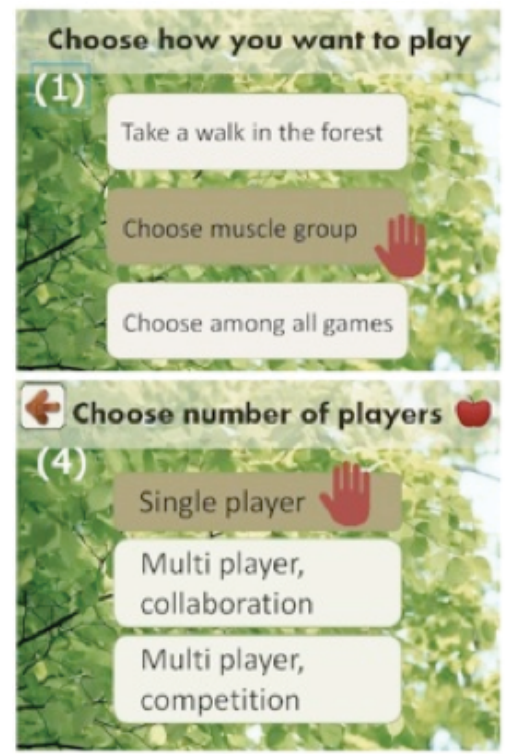

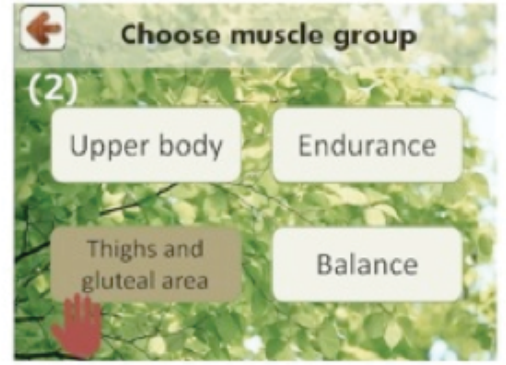

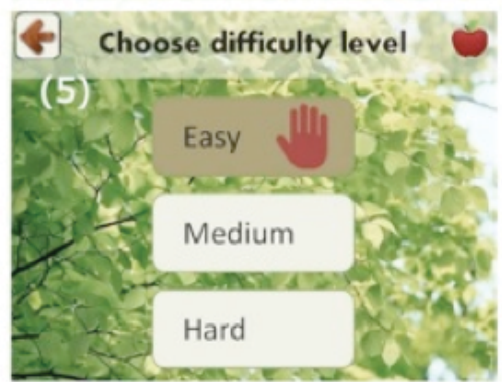

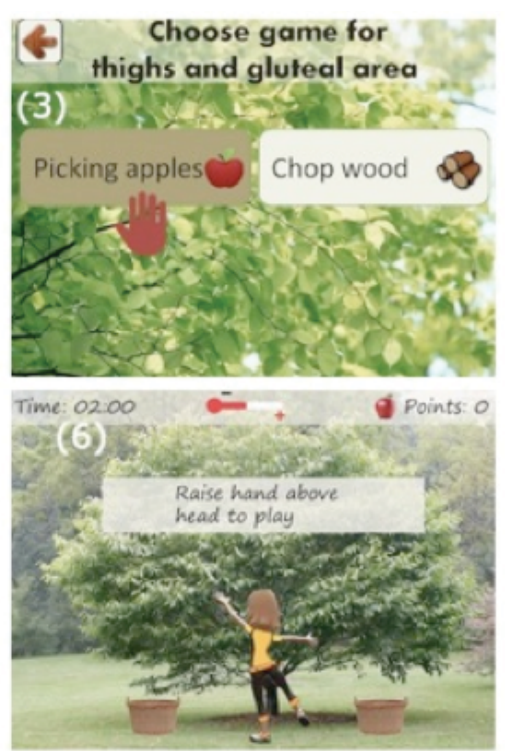

Figure 1. The menu focuses on exercising different muscle groups

performing deep squats (muscle strength). The goal of picking apples is to pick as many ripe apples as possible within a given time. Unripe apples appear on the tree and turn red when they are ripe. If the apple hangs on the tree too long, it rots and falls off the tree. The player gets points from picking red apples, and loses points both for picking unripe apples and from letting an apple rot.

As shown in figure 2, the prototype provides limited information, which is easy to understand for seniors and helps them to focus of obstacles were added, such as (a) jump from rock to rock to get over the river, (b) walk over the log lying across the path, (c) duck under the branch hanging over the path, (d) get over the lake by rowing the boat, (e) balance on the log to get over the river, (f) walk on the rocks lying on the path. As the seniors expressed the idea of doing a quiz in exercise, the design also included quizzes in the nature trail so participants could answer questions while maintaining their balance. 


\subsection{Workshop 2 - Out in Nature}

A second workshop was conducted at the same location as workshop 1 to get the feedback of senior citizens regarding the new exergame prototype. All participants from workshop 1 were invited to participate in the second workshop. Of these, five senior citizens, 3 males and 2 females, participated in the second workshop. A computer and a projector were used to show the concept of the new game. The seniors were also provided with the paper prototypes of the interface. The participants of the workshop were presented with the summary of the issues that were identified in the first workshop and the interface of the new game "Out in Nature". Different sections of the prototype were displayed.

\subsection{Findings from workshop 2}

The participants of the workshop generally liked that the exergame design of 'out in nature' had a familiar environment. However, they were skeptical to including quizzes in the nature trail, because that would take focus away from the physical tasks. One participant stated, Answering the questions in the quiz will become some kind of test on how good you are, and that is not how I have understood the point of these games. They liked that there were different difficulty levels in the game and one participant stated, I think that it is an advantage that everyone starts at the easy level, and the more confident you get the harder it gets. I think that is a good way to be controlled. However, the participants wished clearer description of what was required for different difficulty levels. They generally liked the idea of having a nature trail to play an exergame, which would be entertaining and fruitful and at the same time increase their physical activity level. The participants expressed that the design of the new game was simple to understand as it only showed limited information.

Regarding methodological considerations of the study, it was challenging to present an interactive exergame with a paper prototype for such an inexperienced group, and the participants had little feedback and comments on the game design. For music, the seniors were curious about what kind of music the games would get, I am wondering, about the atmosphere and environment, when I am balancing there [on the log], will I hear the sound of water?

\section{CONCLUSION}

The evaluation of three commercial exergames and presentation of a new exergame provided some important lessons for the design of exergames for senior citizens. This study demonstrates the need to design exergames for seniors with simplified interfaces. First of all, it is important to show only limited information on the screen. Seniors prefer to focus on a single activity in the game instead of doing multiple activities such as physical activity and a quiz at the same time. Secondly, the exergame should provide the objective of the game at the start so that seniors are aware of the activities and their effect. Thirdly, special focus should be given to progress and learning. The progress and learning for exergames might be slower for seniors when comparing it with a younger generation. Lastly, appropriate music should be provided in accordance with seniors' choice. These are important aspects to take into consideration for exergames to be a relevant tool for senior citizens to train their physical function and prevent falls.

The study provides a prototype that gives an example of how a simple interface can be developed which can focus on different training balance and strength for seniors. Designing the game with different exercises would allow seniors to train different muscles and enjoy the game at the same time.

\section{ACKNOWLEDGEMENTS}

The research leading to these results has received funding from the European Union Seventh Framework Programme (FP7/20072013) under grant agreement FARSEEING $n^{\circ} 288940$. FARSEEING is a thematic network focusing on the issue of promoting healthy, independent living for older adults. The authors thank the Departments of Telematics and of Computer and Information Science, NTNU, who contributed equipment needed for the workshop.

\section{REFERENCES}

[1] Factbox on video game industry, Retreived November 14, 2013 from

http://in.reuters.com/article/2013/06/10/gameshow-eidINDEE9590DW20130610,

[2] Billis, A. S., Konstantinidis, E. I., Mouzakidis, C., Tsolaki, M. N., Pappas, C., \& Bamidis, P.D. 2010. A game-like interface for training seniors' dynamic balance and coordination. In Proceedings of IFMBE, Springer, Volume 29, pp 691-694.

[3] Gillespie, L.D., Robertson, MC., Gillespie, Sherrington, C., Gates, S., Clemson, L.M., Lamb, S.E. 2012. Interventions for preventing falls in older people living in the community. Cochrane Database Syst Rev, 9(11).

[4] Rosenberg, D., Depp, C. A., Vahia, I. V., Reichstadt, J., Palmer, B. W., Kerr, J., Jeste, D. V. 2010. Exergames for subsyndromal depression in older adults: A pilot study of a novel intervention. American Journal of Geriatric Psychiatry, 18(3), 221-226

[5] Jung, Y., Li, K. J., Janissa, N. S., Gladys, W. L. C., \& Lee, K. M. 2009. Games for a better life: effects of playing Wii games on the well-being of seniors in a long-term care facility, in Proceedings of the Sixth Australasian Conference on Interactive Entertainment. 2009, ACM: Australia. p. 1-6.

[6] Schoene, D., Lord, S. R., Delbaere, K., Severino, C., Davies, T. A., \& Smith, S.T. 2013. A Randomized Controlled Pilot Study of Home-Based Step Training in Older People Using Videogame Technology. PLoS ONE, 8(3).

[7] Proffitt, R. and B. Lange. 2012.User Centered Design and Development of a Game for Exercise in Older Adults. International Journal of Technology, Knowledge \& Society. $8(5)$

[8] Gerling, K. M., Schild, J., \& Masuch, M. 2010. Exergame design for elderly users: The case study of SilverBalance, in Proceedings of the 7th International Conference on Advances in Computer Entertainment Technology. 2010, ACM: Taipei, Taiwan. p. 66-69.

[9] Jongman, V., Lamoth, C.JC., Van, H., Caljouw, S.R. 2012 Postural control of elderly: moving to predictable and unpredictable targets. Studies in health technology and informatics, 2012. 181: p. 93-97.

[10] Houde, S., \& Hill, C. 1997. What do prototypes prototype. Handbook of human-computer interaction, 2, 367-381 